\title{
7. APPLYING THE MODEL OF A NORMAL LOGARITHMIC SPIRAL TO GALAXIES
}

\author{
R. M. DZIG VASHVILI and T. M. BORCHKHADZE \\ Abastumani Astrophysical Observatory, Abastumani, Georgia, U.S.S.R.
}

\begin{abstract}
The characteristic $\mu$ angles for the spirals of 6 multi-arm galaxies (NGC 1232, 5247, 4303, $4321,3938,3184)$ have been determined. It has been found that they vary along spiral arms within a rather large interval, with mean square deviations ranging from $4^{\circ}$ to $20^{\circ}$. The mean $\mu$ for each arm deviates in a relatively small degree from the mean $\mu$ of the whole galaxy.

The authors apply the normal logarithmic spiral model in estimating the number of arms in our Galaxy. On the basis of this model the number of spiral arms for the above galaxies have been determined and compared with the actual number. It is shown that the normal logarithmic spiral model is unsuitable for our purposes. The number of spiral arms calculated in this way is not reliable.
\end{abstract}

